\title{
Dark and Light triad: the relationship between personality traits and addiction to mobile phones, video games, and the internet
}

Claudio J. Mejía-Suazo', Miguel Landa-Blanco², Gliver Aarón Mejía-Suazo², Carlos A. Martínez-Martínez ${ }^{3}$

${ }^{1}$ School of Biology, Faculty of Sciences, National Autonomous University of Honduras, Honduras

${ }^{2}$ School of Psychological Sciences, Faculty of Social Sciences, National Autonomous University of Honduras, Honduras

${ }^{3}$ Faculty of Medical Sciences, National Autonomous University of Honduras, Honduras

*Correspondence: Claudio.mejia@unah.edu.hn

\begin{abstract}
The present study analyzed the effects of Light and Dark Triad traits scores on Internet Gaming Disorder, intrapersonal and interpersonal conflicts related to internet consumption, conflicts, and communicational emotional usage related to mobile phones. Light Triad traits include Faith in Humanity, Humanism, and Kantianism. Dark Triad traits include Machiavellianism, Narcissism, and Psychopathy. The study used a quantitative approach with a non-experimental design. The sample consisted of 450 adults of the Honduran population, of which $55.33 \%$ were women, and $44.67 \%$ were men, with an average mean age of 25.52 years $(S D=6.79)$. Results indicate that Machiavellianism and Psychopathy scores have significant effects over Internet Gaming Disorder scores. Intrapersonal conflicts related to internet consumption were negatively affected by Narcissism scores and positively affected by Machiavellianism. Psychopathy scores explained interpersonal conflicts related to internet consumption scores. On the other hand, conflicts related to mobile phone usage were negatively affected by Narcissism and Kantianism, while Machiavellianism had positive effects. Finally, communicational emotional usage related to mobile phones was significantly affected by Machiavellianism. These results are discussed according to their implications for the behavioral sciences.
\end{abstract}

Keywords: Dark Triad, Light Triad, behavioral addiction, mobile phones addiction, video games addiction, Internet addiction. 


\section{Introduction}

\section{Dark Triad and Light Triad}

In recent years, the study of personality traits in adult populations has received increased attention from the scientific community. For instance, there is a particular interest in understanding the characteristics of people that, regardless of exhibiting a psychopathic profile, are successfully occupying essential roles in society (Molinuevo, et al., 2017). In this sense, Paulhus \& Williams (2002) have described a combination of "dark" personality traits related to and characterized by offensive behaviors from a social standpoint. These personality traits are not within the clinical or forensic range and are classified as "subclinical".

Paulhus \& Williams (2002) initially used the term "Dark Triad" of personality (DT) to encapsulate the unique combination of attributes including Machiavellianism, Narcissism, and Psychopathy. Even though these form part of the Dark Triad of personality, they each have distinct characteristics. For instance, Machiavellistic personalities use manipulation to exploit others, narcissistic personalities fantasize with power and grandiosity, and psychopathic personalities lack empathy and remorse and tend to have impulsive behavior in search of emotions (González, Garita-Campos, \& Godoy-Izquierdo, 2018). According to Paulhus (2014), people with dark personality traits can develop adaptative behaviors in specific environments.

On the other hand, the Light Triad (LT) of personality is a contemporary measure that surged to explain the inquiries on human cognition and human conduct. The LT, contrary to the DT, focuses on positive personality traits that lead to personal growth in people. The Light Triad of personality has three elements: Kantianism, Humanism, and Faith in Humanity. The first element refers to the idea that human beings should be considered an end in themselves, rather than being valued for their utilitarian potential (Kaufman S., Yaden, Hyde, \& Tsukayama, 2019). Humanism is about recognizing that human beings have worth and dignity (Kaufman S., Yaden, Hyde, \& Tsukayama, 2019). Lastly, Faith in Humanity is the belief that benevolence is a fundamental element present in all human beings (Kaufman S. , Yaden, Hyde, \& Tsukayama, 2019). Based on Kaufman, Yaden, Hyde, \& Tsukayama (2019) proposals, every person expresses dark and light personality traits throughout their lives.

Nevertheless, these traits can be altered by every subject's environment, experiences that generate positive and negative emotions and reactions on a human being. The evolution of technology, for example, has served as a platform for specific behavioral addictions. Some of the most known contemporary behavioral addictions are cellphones, video games, and Internet usage (Petry et al. 2018; Derevensky et al. 2019). 


\section{Internet addiction}

Internet addiction refers to the functional deterioration of a person's wellbeing due to excessive and problematic internet usage. Researchers are becoming increasingly interested in this topic given the increase of the dependency that humans have to the Internet (Vondráčková \& Gabrhelík, 2016; Weinstein \& Lejoyeux, 2010). Internet addiction is characterized by a strong urge to stay permanently connected to the internet, paired with a feeling of anxiety and anguish when disconnected. One of the explanations given to the excessive and uncontrolled use of the internet is that internet addicts need to escape their problems and reality (Weinstein \& Lejoyeux, 2010). In addition, internet addicts suffer from physical and psychological issues such as loneliness, low self-esteem, lack of sleep, fatigue, anxiety, and depression; taking a dangerous toll on their personal, academic, and professional lives (Kawabe et al. 2016; Cheng et al. 2018).

The misuse of the internet can have adverse effects. However, when utilized in a sensible and controlled manner, the internet can potentiate personal, academic, and professional success. The internet can further facilitate interaction with other people and cultures, provide information and knowledge, accelerate economic development, and even access psychological intervention online (Bisen and Deshpande, 2018).

\section{Mobile phones addiction}

The uses for cellphones have changed drastically during the last decades; these were used solely to communicate through calls or text messages. Currently, cellphones are the center for many other uses like photography, videogames, music, voice recording, etc. Mobile phones are also connected to the internet, which allows interactions with people worldwide through social media platforms like Instagram, Facebook, WhatsApp, Twitter, and more. Consequently, mobile phones have become one of the most used technologies for information and communication worldwide (Lopez-Fernandez et al. 2017., Sahu et al. 2019).

The multiple benefits of mobile phones are evident and have become a fundamental element of daily human life. However, the disproportionate use of mobile phones can have dangerous effects. Excessive cellphone use can cause the reduction of physical activity, sleep disturbances, depression, psychological morbidity, and ultimately develop into dependency and addictive use, producing side effects similar to those in people who abuse psychostimulants (loss of control, desire, abstinence, relapse). Evidence has shown that the addiction to mobile phones can cause depression, loss, and isolation when experiencing withdrawal, leading to low performance both in personal and professional aspects (Lopez-Fernandez et al. 2017., Sahu et al. 
2019). These findings have led the World Health Organization to consider the excessive use of mobile phones as a public health concern (WHO, 2015).

\section{Video games addiction}

Gaming addictions refer to recurrent dysfunctional behavioral patterns related to excessive video game consumption, which results in a significant deterioration in personal, social, and occupational wellbeing (World Health Organization, 2018). Gaming addiction has also been compared to substance use disorders since the brain reacts similarly when playing a competitionlike video game and when using psychostimulant drugs (Gros, Debue, Lete, \& van de Leemput, 2020).

Internet gaming disorder prevalence has been identified in a significant proportion of the population (Feng, Ramo, Chan, \& Bourgeois, 2017). It has been documented that males report more problems related to video gaming than females, and young age has been considered a strong predictor for problematic use of video games (Wittek, et al., 2016). The personality traits most commonly associated with gaming addiction have been neuroticism, aggression, hostility, and sensation-seeking.

Video game addiction can lead to several negative psychological consequences: neglecting responsibilities, reduced quality time with loved ones, and sleep alterations. In extreme cases, these individuals may present physical problems such as epileptic seizures, auditory hallucinations, and tenosynovitis. Therefore, the study and intervention of video game addictions have become increasingly important among the general population (Griffiths, Kuss, \& King, 2012). However, not everything is negative in regards to video games. Studies have shown that playing violent video games can be associated with increased visuospatial cognition (Ferguson, 2017), improved mood, and essential prosocial skills when the games have cooperative or supportive goals (Granic, Lobel, \& Engels, 2014). They also have potential value in improving self-esteem, supporting psychotherapeutic treatment, and conflict resolution (Primack, et al., 2012).

\section{Purpose of the study}

Considering all the above, the present study pretends to analyze the effects of Dark Triad traits (as risk factors), and Light Triad traits (as protective factors), regarding Internet Gaming Disorder, intrapersonal and interpersonal conflicts related to internet consumption, conflicts, and communicational-emotional usage related to mobile phones. We believe our study can increase 
the theoretical knowledge regarding behavioral addictions in general, especially within the LatinAmerican context, given that there is little to no information on the matter in this region.

\section{Materials and Methods}

\section{Data collection techniques}

\section{Questionnaire of Experiences Related to the Internet (QERI)}

The Questionnaire of Experiences Related to the Internet (QERI) consists of 10 items distributed in two factors: intrapersonal conflicts $(\alpha=0.74)$ and interpersonal conflicts $(\alpha=0.75)$ (Beranuy-Fargues, Chamarro-Lusar, Graner-Jordania, \& Carbonell-Sánchez, 2009). The scale uses a Likert-type response set of 4 points $(1=$ never, $2=$ almost never, $3=$ almost always, 4=always); higher scores indicate a higher prevalence of internet-related conflicts.

\section{Questionnaire of Experiences Related to the Mobile Phone (QERMP)}

The Questionnaire of Experiences Related to the Mobile Phone (QERMP) consists of 10 items distributed in two factors: conflicts $(\alpha=0.81)$ and communicational/emotional use $(\alpha=0.75)$ (Beranuy-Fargues, Chamarro-Lusar, Graner-Jordania, \& Carbonell-Sánchez, 2009). The scale uses a Likert-type response set of 4 points $(1=$ never, $2=$ almost never, $3=a$ almost always, 4=always); higher scores indicate a higher prevalence of mobile phone experiences.

\section{Internet Gaming Disorder Scale-Short Form (IGDS9-SF)}

The Internet Gaming Disorder Scale-Short Form (IGDS9-SF) consists of 9 items based upon the Diagnostic and Statistical Manual of Mental Disorders fifth edition (DSM-5) criteria for Internet Gaming Disorder. The IGDS9-SF uses a five-point Likert-type response set $(1=$ never, $2=$ rarely, $3=$ sometimes, $4=$ often, $5=$ very often $)$. The scale possesses adequate reliability $(\alpha=0.85)$ and validity (Beranuy, et al., 2020). Higher scores indicate a higher prevalence of internet gaming disorder symptoms.

\section{Short Dark Triad (SD3)}

The Short Dark Triad (SD-3) measures Machiavellianism ( $\alpha=0.76)$, Narcissism $(\alpha=0.78)$, and psychopathic traits $(\alpha=0.73)$. The SD-3 consists of 27 items, with a five-point Likert-type response set $(1=$ disagree strongly, $2=$ disagree, $3=$ neither agree nor disagree, $4=$ agree, $5=$ agree strongly). The SD-3 has also been proven to be a valid measure of dark triad traits (Jones \& Paulhus, 2013). 


\section{Light Triad Scale (LTS)}

The Light Triad Scale (LTS) is a 12-item questionnaire that measures Faith in Humanity $(\alpha=0.80)$, Humanism $(\alpha=0.76)$, and Kantianism $(\alpha=0.67)$, with a total internal consistency of 0.84 , as measured through Cronbach's alpha. The responses use a Likert-type format with 5 points ( $1=$ disagree strongly, $2=$ disagree, $3=$ neither agree nor disagree, $4=$ agree, $5=$ agree strongly). The LTS is considered a valid measurement of light triad traits (Kaufman S. B., Yaden, Hyde, \& Tsukayama, 2019).

\section{Demographic questionnaire}

The demographic questionnaire collected data regarding the respondent's sex (male $=0$; female=1), age, country of residence, a self-reported measure of videogame consumption (hours per day), and mobile phone usage (hours per day).

\section{Sample}

The sample consisted of 450 respondents selected through a non-probabilistic approach. The questionnaires were distributed online via social media and snowball sampling. The inclusion criteria were: 1) being 18 years or older, 2) currently living in Honduras, 3) agreeing to the informed consent. The sample included 259 women (55.33\%) and 201 men (44.67\%); the respondent's mean age was of 25.52 years $(S D=6.79$; minimum age $=18$; maximum age $=56)$. On average, the sample reported playing video games 5.03 hours a day $(S D=4.34)$ and using the mobile phone for 8.25 hours a day $(S D=4.69)$, see Table 1 .

Table 1

Participant's sex and age comparisons

\begin{tabular}{lcccccc}
\hline Group & $n$ & Mean & SD & $t$ & $d f$ & $p$ \\
\hline Female & 249 & 25.048 & 7.132 & \multirow{2}{*}{1.644} & \multirow{2}{*}{448} & 0.101 \\
Male & 201 & 26.104 & 6.304 & & & \\
\hline
\end{tabular}

Note. Males are coded as 0 , females as 1 . Age does not vary significantly between male and female participants.

\section{Ethical considerations}

Informed consent was included at the beginning of each questionnaire. It stated the purpose of the study, a confidentiality statement, and the researcher's contact information. 


\section{Data analysis}

First, total mean scores and standard deviations were determined for each subscale included in the study. An internal consistency analysis was also made through Cronbach's alpha. Then, linear regression models were used to determine the relationship between variables. Specifically, independent variables included: Internet Gaming Disorder scores, Intrapersonal and Interpersonal conflicts related to internet consumption, Communicational Emotional Usage, and Conflicts related to mobile phone usage. Dependent variables included: Narcissism, Machiavellianism, Psychopathy, Humanism, Kantianism, and Faith in Humanity. Determination coefficients $\left(r^{2}\right)$, post-hoc power, and effect sizes $\left(f^{2}\right)$ were calculated for each model. Effect sizes were classified according to Cohen's recommendations: $f^{2}=0.02$ is classified as small, $f^{2}=0.15$ as a medium, and $f^{2}=0.35$ as large (Cohen, 1992). All significance was tested at a $95 \%$ confidence level.

\section{Results}

General description of Dark Triad subscales indicates that Narcissism traits have the highest scores $(M=2.90 ; S D=0.60)$, followed by Machiavellianism $(M=2.74 ; S D=0.71)$ and Psychopathy ( $M=2.01 ; S D=0.74)$. On the other hand, Humanism scores $(M=3.42 ; S D=0.81)$ were the highest among the Light Triad traits, followed by Kantianism $(M=3.87 ; S D=0.74)$ and Faith in Humanity $(M=3.42 ; S D=0.81)$.

The average score in the Internet Gaming Disorder Scale-Short Form was 1.65 $(S D=0.81)$. In the Questionnaire of Experiences Related to Internet $(M=2.13 ; S D=0.49)$, Intrapersonal Conflicts $(M=2.35 ; S D=0.58)$ were more prevalent than interpersonal conflicts $(M=1.91 ; S D=0.55)$. Meanwhile, in the Questionnaire of Experiences Related to Mobile Phone $(M=1.95 ; S D=0.50)$, the Communicational Emotional Usage subscale had higher mean scores $(M=2.16 ; S D=0.54)$ than the Conflicts subscale $(M=1.69 ; S D=0.59)$. The correlation between variables is presented in Table 2.

Table 2

Correlation between variables included in the study

\begin{tabular}{|c|c|c|c|c|c|c|c|c|c|c|c|c|c|}
\hline Variable & 1 & 2 & 3 & 4 & 5 & 6 & 7 & 8 & 9 & 10 & 11 & 12 & 13 \\
\hline 1. Narcissism & - & & & & & & & & & & & & \\
\hline $\begin{array}{l}2 . \\
\text { Machiavellianism }\end{array}$ & $\begin{array}{r}0.23 \\
* * *\end{array}$ & - & & & & & & & & & & & \\
\hline 3. Psychopathy & $\begin{array}{r}0.222 \\
* * *\end{array}$ & $\begin{array}{r}0.594 \\
* * *\end{array}$ & - & & & & & & & & & & \\
\hline 4. Humanism & 0.011 & $\begin{array}{r}0.264 \\
* * *\end{array}$ & $\begin{array}{r}- \\
0.258 \\
* * *\end{array}$ & - & & & & & & & & & \\
\hline
\end{tabular}




\begin{tabular}{|c|c|c|c|c|c|c|c|c|c|c|c|c|c|}
\hline $\begin{array}{l}\text { 5. Faith in } \\
\text { humanity }\end{array}$ & 0.042 & $\begin{array}{r}- \\
0.214 \\
* * *\end{array}$ & $\begin{array}{r}- \\
0.236 \\
* * *\end{array}$ & $\begin{array}{r}0.427 \\
* * *\end{array}$ & - & & & & & & & & \\
\hline 6. Kantianism & $\begin{array}{r}- \\
0.118 \\
*\end{array}$ & $\begin{array}{r}- \\
0.174 \\
* * *\end{array}$ & $\begin{array}{r}- \\
0.205 \\
* * *\end{array}$ & $\begin{array}{r}0.303 \\
* * * \\
\end{array}$ & $\begin{array}{r}0.179 \\
* * * \\
\end{array}$ & - & & & & & & & \\
\hline $\begin{array}{l}\text { 7. Internet } \\
\text { Gaming Disorder }\end{array}$ & $\begin{array}{r}0.103 \\
*\end{array}$ & $\begin{array}{r}0.392 \\
* * *\end{array}$ & $\begin{array}{r}0.443 \\
* * *\end{array}$ & $\begin{array}{r}0.14 \overline{-} \\
* *\end{array}$ & $\begin{array}{r}0.096 \\
*\end{array}$ & $\begin{array}{r}- \\
0.161 \\
* * *\end{array}$ & - & & & & & & \\
\hline $\begin{array}{l}\text { 8. Internet- } \\
\text { Intrapersonal } \\
\text { Conflicts }\end{array}$ & 0.003 & $\begin{array}{r}0.34 \\
* * *\end{array}$ & $\begin{array}{r}0.239 \\
* * *\end{array}$ & $\begin{array}{r}0.093 \\
*\end{array}$ & $0.031^{-}$ & $\begin{array}{r}-0.11 \\
*\end{array}$ & $\begin{array}{r}0.355 \\
* * * \\
\end{array}$ & - & & & & & \\
\hline $\begin{array}{l}\text { 9. Internet- } \\
\text { Interpersonal } \\
\text { Conflicts }\end{array}$ & 0.015 & $\begin{array}{r}0.203 \\
* * *\end{array}$ & $\begin{array}{r}0.221 \\
* * *\end{array}$ & $\begin{array}{r}0.113 \\
*\end{array}$ & $0.033^{-}$ & $0.092^{-}$ & $\begin{array}{r}0.326 \\
* * *\end{array}$ & $\begin{array}{r}0.523 \\
* * *\end{array}$ & - & & & & \\
\hline $\begin{array}{l}\text { 10. Internet- } \\
\text { Total }\end{array}$ & 0.009 & $\begin{array}{r}0.313 \\
* * * \\
\end{array}$ & $\begin{array}{r}0.263 \\
* * * \\
\end{array}$ & $\begin{array}{r}0.116 \\
*\end{array}$ & 0.036 & $\begin{array}{r}0.115 \\
*\end{array}$ & $\begin{array}{r}0.39 \\
* * *\end{array}$ & $\begin{array}{r}0.881 \\
* * * \\
\end{array}$ & $\begin{array}{r}0.864 \\
* * * \\
\end{array}$ & - & & & \\
\hline $\begin{array}{l}\text { 11. Mobile } \\
\text { Phone- Conflicts }\end{array}$ & 0.066 & $\begin{array}{r}0.239 \\
* * *\end{array}$ & $\begin{array}{r}0.212 \\
* * *\end{array}$ & $\begin{array}{r}0.119 \\
*\end{array}$ & $0.076^{-}$ & $\begin{array}{r}0.179 \\
* * *\end{array}$ & $\begin{array}{l}0.35 \\
* * *\end{array}$ & $\begin{array}{r}0.481 \\
* * *\end{array}$ & $\begin{array}{r}0.648 \\
* * *\end{array}$ & $\begin{array}{r}0.643 \\
* * * *\end{array}$ & - & & \\
\hline $\begin{array}{l}\text { 12. Mobile } \\
\text { Phone- } \\
\text { Communicational } \\
\text { emotional usage }\end{array}$ & 0.073 & $\begin{array}{r}0.299 \\
* * *\end{array}$ & $\begin{array}{r}0.21 \\
* * *\end{array}$ & 0.032 & 0.085 & 0.066 & $\begin{array}{r}0.326 \\
* * * \\
\end{array}$ & $\begin{array}{r}0.648 \\
* * *\end{array}$ & $\begin{array}{r}0.52 \\
* * * \\
\end{array}$ & $\begin{array}{r}0.672 \\
* * *\end{array}$ & $\begin{array}{r}0.546 \\
* * * \\
\end{array}$ & - & \\
\hline $\begin{array}{l}\text { 13. Mobile } \\
\text { Phone-Total }\end{array}$ & 0.001 & $\begin{array}{r}0.304 \\
* * * \\
\end{array}$ & $\begin{array}{r}0.24 \\
* * * \\
\end{array}$ & 0.088 & 0.091 & $\begin{array}{r}0.142 \\
* *\end{array}$ & $\begin{array}{r}0.385 \\
* * * \\
\end{array}$ & $\begin{array}{r}0.637 \\
* * * \\
\end{array}$ & $\begin{array}{r}0.668 \\
* * * \\
\end{array}$ & $\begin{array}{r}0.747 \\
\quad * * * \\
\end{array}$ & $\begin{array}{r}0.891 \\
* * * *\end{array}$ & $\begin{array}{r}0.866 \\
* * *\end{array}$ & - \\
\hline 14. Age & 0.008 & $\begin{array}{r}0.112 \\
* \\
\end{array}$ & $\begin{array}{r}- \\
0.107 \\
*\end{array}$ & 0.032 & 0.068 & 0.054 & $\begin{array}{r}- \\
0.115 \\
*\end{array}$ & $\begin{array}{r}- \\
0.159 \\
* * * \\
\end{array}$ & $\begin{array}{r}- \\
0.183 \\
* * *\end{array}$ & $\begin{array}{r}- \\
0.196 \\
* * *\end{array}$ & $\begin{array}{r}-0.221 \\
* * * \\
\end{array}$ & $\begin{array}{r}-0.205 \\
* * * \\
\end{array}$ & $\begin{array}{r}0.243 \\
* * * \\
\end{array}$ \\
\hline
\end{tabular}

Note. Correlation coefficients were calculated with Pearson's $r$.

$* \mathrm{p}<.05, * * \mathrm{p}<.01, * * * \mathrm{p}<.001$

Regression models were used to determine the influence of sex, age, Light and Dark Triad traits over the scores of Internet Gaming Disorder, intrapersonal and interpersonal conflicts related to internet consumption, conflicts, and communicational emotional usage related to mobile phones. In this sense, the model significantly explains Internet Gaming Disorder scores $\left(r^{2}=0.26\right)$, with Age and being female having negative effects, while Machiavellianism and Psychopathy scores having significant positive effects. The model achieved a large effect size, $f^{2}=.356$.

Intrapersonal conflicts related to internet consumption were significantly explained by the model, with a medium effect size $\left(r^{2}=0.15 ; f^{2}=.173\right)$. Narcissism scores have negative effects over such intrapersonal conflicts, while Machiavellianism scores are positively related to such outcomes. On the other hand, the model accounted for $9 \%$ of interpersonal conflicts related to internet consumption scores $\left(r^{2}=0.090 ; f^{2}=0.100\right)$; this effect size is classified as small. With Age having significant negative effects, and Psychopathy being positively related to interpersonal conflicts. 
Conflicts related to mobile phone usage were also significantly explained by the model $\left(r^{2}=0.14 ; f^{2}=.158\right)$; the achieved effect size is classified as medium. Narcissism, Kantianism and Age, are inversely related to such conflicts, while Machiavellianism has positive effects. Finally, the proposed model significantly predicts communicational emotional usage related to mobile phones $\left(r^{2}=0.14 ; f^{2}=157\right)$. With Age being a significant negative predictor, while being female and Machiavellianism being positively related to communicational emotional usage. The achieved effect size is classified as medium, see Table 3 .

Table 3

Regression models explaining behavioral addictions

\begin{tabular}{|c|c|c|c|c|c|c|c|c|c|c|}
\hline \multirow{2}{*}{ Outcome } & \multirow{2}{*}{ Predictor } & \multirow{2}{*}{$\begin{array}{c}\text { Unstandardi } \\
\text { zed }\end{array}$} & \multirow{2}{*}{ SE } & \multirow{2}{*}{$\begin{array}{l}\text { Standardi } \\
\text { zed }\end{array}$} & \multirow{2}{*}{$p$} & \multicolumn{2}{|c|}{$95 \% \mathrm{CI}$} & \multirow{2}{*}{$r^{2}$} & \multirow{2}{*}{$f^{2}$} & \multirow{2}{*}{$F$} \\
\hline & & & & & & $L L$ & $U L$ & & & \\
\hline \multirow{9}{*}{$\begin{array}{l}\text { Internet } \\
\text { Gaming } \\
\text { Disorder }\end{array}$} & (Intercept) & 1.111 & $\begin{array}{r}0.38 \\
1\end{array}$ & & $\begin{array}{r}0.00 \\
4\end{array}$ & $\begin{array}{r}0.36 \\
3 \\
\end{array}$ & 1.86 & \multirow{9}{*}{$\begin{array}{c}0.2 \\
6\end{array}$} & \multirow{9}{*}{$\begin{array}{c}.356 \\
(>0.9 \\
9)\end{array}$} & \multirow{9}{*}{$\begin{array}{c}19.697 \\
(\mathrm{p}<0.00 \\
1)\end{array}$} \\
\hline & Age & -0.01 & $\begin{array}{r}0.00 \\
5 \\
\end{array}$ & -0.083 & $\begin{array}{r}0.04 \\
9 \\
\end{array}$ & 0.02 & 0. & & & \\
\hline & Sex & -0.323 & $\begin{array}{r}0.07 \\
3 \\
\end{array}$ & -0.199 & $\begin{array}{l}<.0 \\
01\end{array}$ & $\begin{array}{r}0.46 \\
7 \\
\end{array}$ & $0.18^{-}$ & & & \\
\hline & Narcissism & -0.021 & $\begin{array}{r}0.05 \\
8\end{array}$ & -0.016 & $\begin{array}{r}0.71 \\
6\end{array}$ & $\begin{array}{r}0.13 \\
5\end{array}$ & $\begin{array}{r}0.09 \\
3\end{array}$ & & & \\
\hline & $\begin{array}{l}\text { Machiavellian } \\
\text { ism }\end{array}$ & 0.191 & 0.06 & 0.167 & $\begin{array}{r}0.00 \\
2\end{array}$ & $\begin{array}{r}0.07 \\
4 \\
\end{array}$ & $\begin{array}{r}0.30 \\
9\end{array}$ & & & \\
\hline & Psychopathy & 0.292 & $\begin{array}{r}0.05 \\
9\end{array}$ & 0.266 & $\begin{array}{l}<.0 \\
01\end{array}$ & $\begin{array}{r}0.17 \\
7\end{array}$ & $\begin{array}{r}0.40 \\
8\end{array}$ & & & \\
\hline & $\begin{array}{l}\text { Faith in } \\
\text { humanity }\end{array}$ & 0.029 & $\begin{array}{r}0.04 \\
6 \\
\end{array}$ & 0.029 & $\begin{array}{r}0.53 \\
2 \\
\end{array}$ & $\begin{array}{r}0.06 \\
1 \\
\end{array}$ & $\begin{array}{r}0.11 \\
9 \\
\end{array}$ & & & \\
\hline & Humanism & -0.002 & $\begin{array}{r}0.05 \\
6 \\
\end{array}$ & -0.001 & $\begin{array}{r}0.97 \\
8\end{array}$ & $\begin{array}{r}0.11 \\
1\end{array}$ & $\begin{array}{r}0.10 \\
8 \\
\end{array}$ & & & \\
\hline & Kantianism & -0.045 & $\begin{array}{r}0.04 \\
8 \\
\end{array}$ & -0.041 & $\begin{array}{r}0.35 \\
2 \\
\end{array}$ & $\begin{array}{r}- \\
0.14\end{array}$ & 0.05 & & & \\
\hline \multirow{7}{*}{$\begin{array}{l}\text { Internet- } \\
\text { Intrapersonal } \\
\text { Conflicts }\end{array}$} & (Intercept) & 2.025 & $\begin{array}{r}0.29 \\
4 \\
\end{array}$ & & $\begin{array}{l}<.0 \\
01\end{array}$ & $\begin{array}{r}1.44 \\
7 \\
\end{array}$ & $\begin{array}{r}2.60 \\
2 \\
\end{array}$ & \multirow{7}{*}{$\begin{array}{c}0.1 \\
5\end{array}$} & \multirow{7}{*}{$\begin{array}{c}0.173 \\
(>0.9 \\
9)\end{array}$} & \multirow{7}{*}{$\begin{array}{c}9.564 \\
(\mathrm{p}<0.00 \\
1)\end{array}$} \\
\hline & Age & -0.01 & $\begin{array}{r}0.00 \\
4\end{array}$ & -0.112 & $\begin{array}{r}0.01 \\
3\end{array}$ & $\begin{array}{r}0.01 \\
7\end{array}$ & $\begin{array}{r}0.00 \\
2\end{array}$ & & & \\
\hline & Sex & 0.063 & $\begin{array}{r}0.05 \\
6\end{array}$ & 0.054 & $\begin{array}{r}0.26 \\
4\end{array}$ & $\begin{array}{r}0.04 \\
8\end{array}$ & $\begin{array}{r}0.17 \\
4\end{array}$ & & & \\
\hline & Narcissism & -0.091 & $\begin{array}{r}0.04 \\
5\end{array}$ & -0.094 & $\begin{array}{r}0.04 \\
2\end{array}$ & $\begin{array}{r}0.17 \\
9\end{array}$ & $\begin{array}{r}0.00 \\
3\end{array}$ & & & \\
\hline & $\begin{array}{l}\text { Machiavellian } \\
\text { ism }\end{array}$ & 0.263 & $\begin{array}{r}0.04 \\
6 \\
\end{array}$ & 0.321 & $\begin{array}{l}<.0 \\
01\end{array}$ & $\begin{array}{r}0.17 \\
2 \\
\end{array}$ & $\begin{array}{r}0.35 \\
4 \\
\end{array}$ & & & \\
\hline & Psychopathy & 0.062 & $\begin{array}{r}0.04 \\
5\end{array}$ & 0.078 & $\begin{array}{r}0.17 \\
5\end{array}$ & $\begin{array}{r}0.02 \\
8\end{array}$ & $\begin{array}{r}0.15 \\
1\end{array}$ & & & \\
\hline & $\begin{array}{l}\text { Faith in } \\
\text { humanity }\end{array}$ & 0.059 & $\begin{array}{r}0.03 \\
5\end{array}$ & 0.083 & $\begin{array}{r}0.09 \\
5\end{array}$ & $0 . \overline{-}$ & $\begin{array}{r}0.12 \\
9\end{array}$ & & & \\
\hline
\end{tabular}




\begin{tabular}{|c|c|c|c|c|c|c|c|c|c|c|}
\hline & Humanism & -0.013 & $\begin{array}{r}0.04 \\
3 \\
\end{array}$ & -0.016 & $\begin{array}{r}0.75 \\
9 \\
\end{array}$ & $\begin{array}{r}0.09 \\
7\end{array}$ & $\begin{array}{r}0.07 \\
1 \\
\end{array}$ & & & \\
\hline & Kantianism & -0.051 & $\begin{array}{r}0.03 \\
7 \\
\end{array}$ & -0.064 & $\begin{array}{r}0.17 \\
5 \\
\end{array}$ & $\begin{array}{r}0.12 \\
4 \\
\end{array}$ & $\begin{array}{r}0.02 \\
3 \\
\end{array}$ & & & \\
\hline \multirow{9}{*}{$\begin{array}{l}\text { Internet- } \\
\text { Interpersonal } \\
\text { Conflicts }\end{array}$} & (Intercept) & 2.137 & $\begin{array}{r}0.28 \\
5 \\
\end{array}$ & & $\begin{array}{l}<.0 \\
01 \\
\end{array}$ & $\begin{array}{r}1.57 \\
7 \\
\end{array}$ & $\begin{array}{r}2.69 \\
8 \\
\end{array}$ & \multirow{9}{*}{$\begin{array}{c}0.0 \\
9\end{array}$} & \multirow{9}{*}{$\begin{array}{l}0.100 \\
(>0.9 \\
9)\end{array}$} & \multirow{9}{*}{$\begin{array}{c}5.510 \\
(\mathrm{p}<0.00 \\
1)\end{array}$} \\
\hline & Age & -0.013 & $\begin{array}{r}0.00 \\
4 \\
\end{array}$ & -0.165 & $\begin{array}{l}<.0 \\
01\end{array}$ & $\begin{array}{r}- \\
0.02 \\
1 \\
\end{array}$ & $\begin{array}{r}- \\
0.00 \\
6 \\
\end{array}$ & & & \\
\hline & Sex & -0.028 & $\begin{array}{r}0.05 \\
5\end{array}$ & -0.025 & $\begin{array}{r}0.61 \\
2\end{array}$ & $\begin{array}{r}0.13 \\
5 \\
\end{array}$ & 0.08 & & & \\
\hline & Narcissism & -0.041 & $\begin{array}{r}0.04 \\
3 \\
\end{array}$ & -0.044 & $\begin{array}{r}0.35 \\
2\end{array}$ & $\begin{array}{r}0.12 \\
6\end{array}$ & $\begin{array}{r}0.04 \\
5\end{array}$ & & & \\
\hline & $\begin{array}{l}\text { Machiavellian } \\
\text { ism }\end{array}$ & 0.073 & $\begin{array}{r}0.04 \\
5 \\
\end{array}$ & 0.094 & $\begin{array}{r}0.10 \\
6\end{array}$ & $\begin{array}{r}0.01 \\
6\end{array}$ & $\begin{array}{r}0.16 \\
1\end{array}$ & & & \\
\hline & Psychopathy & 0.104 & $\begin{array}{r}0.04 \\
4 \\
\end{array}$ & 0.14 & $\begin{array}{r}0.01 \\
9\end{array}$ & $\begin{array}{r}0.01 \\
7 \\
\end{array}$ & 0.19 & & & \\
\hline & $\begin{array}{l}\text { Faith in } \\
\text { humanity }\end{array}$ & 0.048 & $\begin{array}{r}0.03 \\
4 \\
\end{array}$ & 0.072 & $\begin{array}{r}0.16 \\
2 \\
\end{array}$ & $\begin{array}{r}0.01 \\
9 \\
\end{array}$ & $\begin{array}{r}0.11 \\
6 \\
\end{array}$ & & & \\
\hline & Humanism & -0.058 & $\begin{array}{r}0.04 \\
2 \\
\end{array}$ & -0.074 & $\begin{array}{r}0.16 \\
4 \\
\end{array}$ & $0 . \overline{-}$ & $\begin{array}{r}0.02 \\
4 \\
\end{array}$ & & & \\
\hline & Kantianism & -0.021 & $\begin{array}{r}0.03 \\
6\end{array}$ & -0.028 & $\begin{array}{r}0.56 \\
4\end{array}$ & $\begin{array}{r}0.09 \\
2\end{array}$ & 0.05 & & & \\
\hline \multirow{9}{*}{$\begin{array}{l}\text { Mobile } \\
\text { phone- } \\
\text { Conflicts }\end{array}$} & (Intercept) & 2.372 & $\begin{array}{r}0.30 \\
3\end{array}$ & & $\begin{array}{l}<.0 \\
01\end{array}$ & $\begin{array}{r}1.77 \\
8\end{array}$ & $\begin{array}{r}2.96 \\
7\end{array}$ & \multirow{9}{*}{$\begin{array}{c}0.1 \\
4\end{array}$} & \multirow{9}{*}{$\begin{array}{c}.158 \\
(>0.9 \\
9)\end{array}$} & \multirow{9}{*}{$\begin{array}{c}8.740 \\
(\mathrm{p}<0.00 \\
1)\end{array}$} \\
\hline & Age & -0.016 & $\begin{array}{r}0.00 \\
4\end{array}$ & -0.184 & $\begin{array}{l}<.0 \\
01\end{array}$ & $\begin{array}{r}0.02 \\
4\end{array}$ & $\begin{array}{r}0.00 \\
8\end{array}$ & & & \\
\hline & Sex & 0.026 & $\begin{array}{r}0.05 \\
8 \\
\end{array}$ & 0.022 & $\begin{array}{r}0.64 \\
8 \\
\end{array}$ & $\begin{array}{r}0.08 \\
7\end{array}$ & 0.14 & & & \\
\hline & Narcissism & -0.142 & $\begin{array}{r}0.04 \\
6\end{array}$ & -0.143 & $\begin{array}{r}0.00 \\
2\end{array}$ & $\begin{array}{r}0.23 \\
3\end{array}$ & $\begin{array}{r}0.05 \\
1\end{array}$ & & & \\
\hline & $\begin{array}{l}\text { Machiavellian } \\
\text { ism }\end{array}$ & 0.146 & $\begin{array}{r}0.04 \\
8\end{array}$ & 0.174 & $\begin{array}{r}0.00 \\
2\end{array}$ & $\begin{array}{r}0.05 \\
3\end{array}$ & 0.24 & & & \\
\hline & Psychopathy & 0.082 & $\begin{array}{r}0.04 \\
7 \\
\end{array}$ & 0.102 & 0.08 & 0. & $\begin{array}{r}0.17 \\
4 \\
\end{array}$ & & & \\
\hline & $\begin{array}{l}\text { Faith in } \\
\text { humanity }\end{array}$ & 0.029 & $\begin{array}{r}0.03 \\
6\end{array}$ & 0.04 & $\begin{array}{r}0.41 \\
9\end{array}$ & $\begin{array}{r}0.04 \\
2\end{array}$ & $\begin{array}{r}0.10 \\
1\end{array}$ & & & \\
\hline & Humanism & -0.026 & $\begin{array}{r}0.04 \\
4\end{array}$ & -0.031 & $\begin{array}{r}0.55 \\
3 \\
\end{array}$ & $\begin{array}{r}0.11 \\
3 \\
\end{array}$ & $\begin{array}{r}0.06 \\
1 \\
\end{array}$ & & & \\
\hline & Kantianism & -0.11 & $\begin{array}{r}0.03 \\
8 \\
\end{array}$ & -0.137 & $\begin{array}{r}0.00 \\
4\end{array}$ & $\begin{array}{r}0.18 \\
6 \\
\end{array}$ & $\begin{array}{r}0.03 \\
5 \\
\end{array}$ & & & \\
\hline \multirow{2}{*}{$\begin{array}{c}\text { Mobile } \\
\text { Phone- } \\
\text { Communicati } \\
\text { onal }\end{array}$} & (Intercept) & 1.665 & $\begin{array}{r}0.27 \\
5 \\
\end{array}$ & & $\begin{array}{l}<.0 \\
01 \\
\end{array}$ & $\begin{array}{r}1.12 \\
5 \\
\end{array}$ & $\begin{array}{r}2.20 \\
6 \\
\end{array}$ & \multirow{2}{*}{$\begin{array}{c}0.1 \\
4\end{array}$} & \multirow{2}{*}{$\begin{array}{l}0.157 \\
(>0.9 \\
9)\end{array}$} & \multirow{2}{*}{$\begin{array}{c}8.674 \\
(0<0.00 \\
1)\end{array}$} \\
\hline & Age & -0.012 & $\begin{array}{r}0.00 \\
4\end{array}$ & -0.151 & $\begin{array}{l}<.0 \\
01\end{array}$ & $\begin{array}{r}- \\
0.01 \\
9\end{array}$ & $\begin{array}{r}- \\
0.00 \\
5\end{array}$ & & & \\
\hline
\end{tabular}




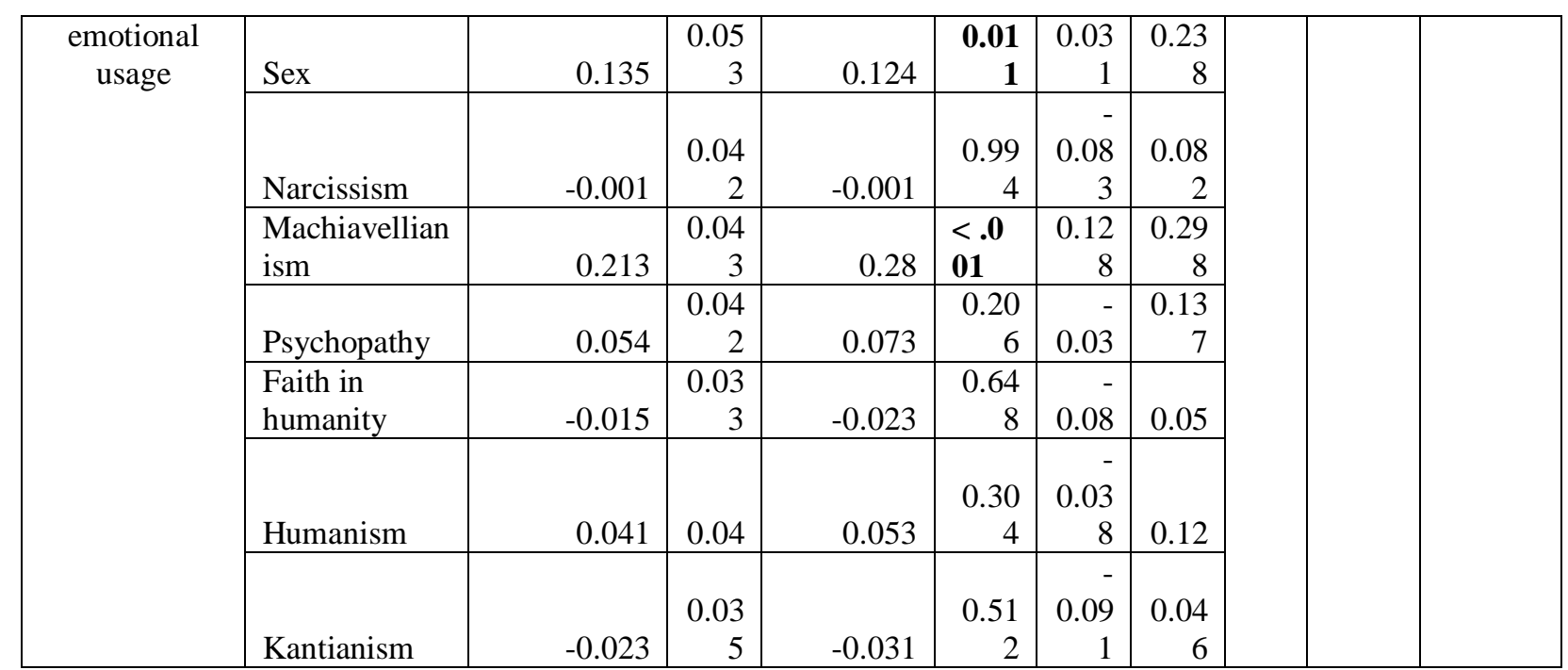

Note. Significant $p$-values are presented in bold letters. Male participants were coded as " 0 ", females as " 1 ".

\section{Discussion}

Our study explored the relationship between the Dark Traits of personality (as a risk factor) and the Light Traits (as a protective factor) regarding mobile phones, video games, and internet addiction. In this sense, our findings suggest that such outcomes were significantly predicted by at least one Dark Triad trait. However, for the most part, Light Traits of personality were not significant predictors of the behavioral addictions included in the study, save for Kantianism, which serves as a protective factor for conflicts related to mobile phone usage.

The above relates to a study made by Tang et al. (2020), who reported that Machiavellianism and Psychopathy were significant predictors of addictive videogame use. These results support our findings since people with addictive behaviors to video games also had high scores of Machiavellianism and Psychopathy. This may relate to the fact that most video games have a reward system that serves as a significant stimulus for people with Machiavellianism and Psychopathic traits. We also found significant differences between men and women, since women showed lower scores of gaming disorder, which can be caused by the harassment received from other male players and a high level of violence and sexuality present in video games (LopezFernandez, Williams, Griffiths, \& Kuss, 2019).

In the case of the addictive behaviors towards the internet, similar results were observed, since people who had interpersonal conflicts related to the addictive use of the internet had a high score of Machiavellianism. On the other hand, higher scores for Narcissism were related to reduced intrapersonal conflict regarding addictive behavior on the internet. Several studies like the one made by Kircaburun y Griffiths (2018), have found that the addictive or problematic use 
of the internet is related to high levels of Machiavellianism. This is probably caused by the fact that people have unlimited access to activities and websites (videogames, online shopping, social media) that encourage them to be online constantly. Additionally, the excessive use of the internet may expose people to an overwhelming amount of information and stimuli available on the web. This can lead to intrapersonal conflicts like difficulties in making decisions, low self-esteem, and depression (Anju, Amandeep, Punia, Punia, \& Garg, 2021). On the other side, people with high psychopath levels showed more interpersonal conflicts related to the excessive use of the internet. This can be caused because people with psychopath traits can have difficulty establishing social relationships (Sindermann, Sariyska, Lachmann, Brand, \& Montag, 2018).

Our findings indicate that higher scores in conflictive use of mobile phones were related to higher Machiavellianism traits. At the same time, we found that high scores of Narcissisms were related to lower conflicts with the addictive use of mobile phones. Although people with predominant Narcissism traits tend to spend more time using their mobile phones (Gökçearslan, Yildiz, Berikan, \& Saritepeci, 2021), previous studies have also reported an inverse relationship between Narcissisms and the addictive behavior to mobile phones (Hussain, Griffiths, \& Sheffield, 2017).

Our study concludes that people with high scores of Kantianism had fewer conflicts with the addictive use of mobile phones. Considering that people with this trait tend not to see other people utilitarianly, it can be inferred that this "protects" them from developing dependence on the mobile phone. It was also found that women use more emotional communication in relation to the addictive behaviors of mobile phones. Women who had higher Machiavellianism scores were also the ones who had more emotional communication through mobile phones. Previous studies have suggested that women are more likely to use mobile phones to interact with others (De-Sola Gutiérrez, Rodríguez de Fonseca, \& Rubio, 2016).

Lastly, an age analysis was made, and it showed that the older the age, the less score for addictive behaviors; this tendency was present in all the variables that were studied. This indicates that young adults are particularly vulnerable to suffer these types of addiction, which is why the public policies of mental health should prioritize this age group. In this sense, our research has theoretical and practical implications. It is the first research that deals with addictive-compulsive behaviors towards the internet, video games, and mobile phones, by considering Dark Triad traits and Light personality traits.

The theoretical contribution of this study lies in the enrichment offered in behavioral addictions, showing how in most cases, there is a direct relationship between the excessive use of these new technologies and the negative traits of personality. This knowledge could help people use this technology more adequately. Being conscious that a person shows high levels of these 
Dark traits could predispose to developing a more significant addiction or dependence. In addition, for the most part, Light personality traits were not a determining factor in protecting from the behavioral addictions included in our study. Therefore, public policy must not underestimate the risks implied by the misuse of technology and addictive potential.

Despite the relevance of our findings, some limitations must be considered. First, the limited sample size and non-probabilistic selection of the participants may limit the generalizability of our results. Second, our study was based upon self-reported measurements, which participants might be biased to respond. More research is needed to corroborate further the evidence presented by our study. Future studies should also focus on the prevalence and predictor of behavioral addictions in low-and-middle-income countries and their corresponding psychosocial interventions. Additionally, further research should evaluate behavioral addictions' effects on mental health indicators like depression, anxiety, somatization, and wellbeing.

\section{Funding}

The authors received no financial support for the research.

\section{Acknowledgments}

The authors would like to thank Lissa M. Flores-Nuñez for assisting in the language adaptation process

\section{Data Availability Statement}

The raw data supporting the conclusions of this article will be made available by the authors without undue reservation.

\section{References}

Anju, A., Amandeep, A., Punia, B. K., Punia, V., \& Garg, N. (2021). Life dissatisfaction among students: exploring the role of intrapersonal conflict, insufficient efforts and academic stress. Rajagiri Management Journal, (ahead-of-print). doi:https://doi.org/10.1108/RAMJ-09-2020-0058

Beranuy, M., Machimbarrena, J., Vega-Osés, A., Carbonell, X., Griffiths, M., Pontes, H., \& González-Cabrera, J. (2020). Spanish Validation of the Internet Gaming Disorder ScaleShort Form (IGDS9-SF): Prevalence and Relationship with Online Gambling and Quality of Life. International Journal of Environmental Research and Public Health, 17(1562). doi:10.3390/ijerph17051562

Beranuy-Fargues, M., Chamarro-Lusar, A., Graner-Jordania, C., \& Carbonell-Sánchez, X. (2009). Validación de dos escalas breves para evaluar la adicción a Internet y el abuso de 
móvil. Psicothema, 21(3), 480-485. Retrieved from http://www.psicothema.com/psicothema.asp?id=3658

Bisen, S., \& Deshpande, Y. (2018). "Understanding internet addiction: a comprehensive review". Mental Health Review Journal, 23(3), 165-184. doi:10.1108 / MHRJ-07-2017-0023

Cheng, Y., Tseng, P., Lin, P., Chen, T., Stubbs, B., Carvalho, A., . . Wu, M. (2018). Internet Addiction and Its Relationship with Suicidal Behaviors: A Meta- Analysis of Multinational Observational Studies. J Clin Psychiatry, 79(4), 17r11761. doi:10.4088/JCP.17r11761

Cohen, J. (1992). A Power Primer. Quantitative Methods In Psychology, 112(1), 155-159. doi:10.1037//0033-2909.112.1.155

Derevensky, J., Hayman, V., \& Lynette, G. (2019). Behavioral Addictions: Excessive Gambling, Gaming, Internet, and Smartphone Use Among Children and Adolescents. Pediatric clinics of North America, 66(6), 1163-1182. doi:10.1016/j.pcl.2019.08.008

De-Sola Gutiérrez, J., Rodríguez de Fonseca, F., \& Rubio, G. (2016). Cell-Phone Addiction: A Review. Front Psychiatr, 7, 1-15. doi:https://doi.org/10.3389/fpsyt.2016.00175

Feng, W., Ramo, D., Chan, S., \& Bourgeois, J. (2017). Internet gaming disorder: Trends in prevalence 1998-2016. Addictive behaviors, 75, 17-24. doi:10.1016/j.addbeh.2017.06.010

Ferguson, C. (2017). The good, the bad and the ugly: a meta-analytic review of positive and negative effects of violent video games. The Psychiatric quarterly, 78(4), 309-316. doi:10.1007/s11126-007-9056-9

Gökçearslan, S., Yildiz, D. H., Berikan, B., \& Saritepeci, M. (2021). Smartphone Addiction, Loneliness, Narcissistic Personality, and Family Belonging Among University Students: A Path Analysis. Social Science Quarterly, 1-18. doi:https://doi.org/10.1111/ssqu.12949

González, J., Garita-Campos, D., \& Godoy-Izquierdo, D. (2018). La triada oscura de la personalidad y sus implicaciones psicológicas en el deporte. Una revisión sistemática. Revista Cuadernos de Psicología del Deporte, 2(18), 191-207. Retrieved from https://revistas.um.es/cpd/article/view/323031/229561

Granic, I., Lobel, A., \& Engels, R. C. (2014). The benefits of playing video games. The American psychologist, 69(1), 66-78. doi:10.1037/a0034857

Griffiths, M., Kuss, D., \& King, D. (2012). Video Game Addiction: Past, Present and Future. Current Psychiatry Research and Reviews, 8(4), 308-318. Retrieved from https://psycnet.apa.org/record/2013-29466-012

Gros, L., Debue, N., Lete, J., \& van de Leemput, C. (2020). Video Game Addiction and Emotional States: Possible Confusion Between Pleasure and Happiness? Frontiers in psychology, 10(2894). doi:https://doi.org/10.3389/fpsyg.2019.02894

Hussain, Z., Griffiths, M. D., \& Sheffield, D. (2017). An investigation into problematic smartphone use: The role of narcissism, anxiety, and personality factors. $J$ Behav Addict, 6(3), 378-386. doi:https://doi.org/10.1556/2006.6.2017.052

Jones, D., \& Paulhus, D. (2013). Introducing the Short Dark Triad (SD3): A Brief Measure of Dark Personality Traits. Assessment, 21(1), 28-41. doi:10.1177/1073191113514105

Kaufman, S. B., Yaden, D. B., Hyde, E., \& Tsukayama, E. (2019). The Light vs. Dark Triad of Personality: Contrasting Two Very Different Profiles of Human Nature. Frontiers in psychology, 10(467). doi:10.3389/fpsyg.2019.00467

Kaufman, S., Yaden, D., Hyde, E., \& Tsukayama, E. (2019). The Light vs. Dark Triad of Personality: Contrasting Two Very Different Profiles of Human Nature. Front. Psychol, 10, 1-26. doi:10.3389/fpsyg.2019.00467

Kawabe, K., Horiuchi, F., Ochi, M., Oka, Y., \& Ueno, S. (2016). Internet addiction: Prevalence and relation with mental states in adolescents. Psychiatry Clin Neurosci, 70(9), 405-12. doi:10.1111/pen.12402

Kircaburun, K., \& Griffiths, M. (2018). The dark side of internet: Preliminary evidence for the associations of dark personality traits with specific online activities and problematic internet use. J Behav Addict, 7(4), 993-1003. doi:10.1556/2006.7.2018.109 
Lopez-Fernandez, O., Kuss, D., Romo, L., Morvan, Y., Kern, L., Graziani, P., . . . Po. (2017). Self-reported dependence on mobile phones in young adults: A European cross-cultural empirical survey. J Behav Addict, 6(2), 168-177. doi:10.1556/2006.6.2017.020

Lopez-Fernandez, O., Williams, A., Griffiths, M., \& Kuss, D. (2019). Female Gaming, Gaming Addiction, and the Role of Women Within Gaming Culture: A Narrative Literature Review. Front Psychiatry, 10, 1-14. doi:https://doi.org/10.3389/fpsyt.2019.00454

Molinuevo, B., Garreta, D., Rafael, T., Martínez, E., Bonillo, A., Requena, A., . . Martínez, J. (2017). La tétrada oscura y la predicción de conducta agresiva, antisocial y de adaptación institucional en jóvenes internados en centros educativos. Generalitat de Catalunya. Cataluña, España: Centre d'Estudis Jurídics i Formació Especialitzada. Retrieved from http://cejfe.gencat.cat/es/recerca/cataleg/crono/2018/tetrada-fosca/

Paulhus, D. (2014). Toward a Taxonomy of Dark Personalities. Current Directions in Psychological Science, 23(6), 421-426. doi:10.1177/0963721414547737

Paulhus, D., \& Williams, K. (2002). The Dark Triad of personality: Narcissism, Machiavellianism, and psychopathy. Journal of Research in Personality, 36(6), 556-563. doi:10.1016/S0092-6566(02)00505-6

Petry, N., Zajac, K., \& Ginley, M. (2018). Behavioral Addictions as Mental Disorders: To Be or Not To Be? Annu Rev Clin Psychol, 7(14), 399-423. doi:10.1146/annurev-clinpsy032816-045120

Primack, B., Carroll, M., McNamara, M., Klem, M., King, B., Rich, M., . . Nayak, S. (2012). Role of video games in improving health-related outcomes: a systematic review. American journal of preventive medicine, 42(6), 630-638. doi:10.1016/j.amepre.2012.02.023

Sahu, M., Gandhi, S., \& Sharma, M. (2019). Mobile Phone Addiction Among Children and Adolescents: A Systematic Review. $J$ Addict Nurs, 30(4), 261-268. doi:10.1097/JAN.0000000000000309

Sindermann, C., Sariyska, R., Lachmann, B., Brand, M., \& Montag, C. (2018). Associations between the dark triad of personality and unspecified/specific forms of Internet-use disorder. J Behav Addict, 985-992. doi:10.1556/2006.7.2018.114

Tang, W. Y., Reer, F., \& Quandt, T. (2020). The interplay of gaming disorder, gaming motivations, and the dark triad. $J$ Behav Addict, 9(2), 1-6. doi:https://doi.org/10.1556/2006.2020.00013

Vondráčková, P., \& Gabrhelík, R. (2016). Prevention of Internet addiction: A systematic review. $J$ Behav Addict, 5(4), 568-579. doi:10.1556/2006.5.2016.085

Weinstein, A., \& Lejoyeux, M. (2010). Internet addiction or excessive internet use. Am J Drug Alcohol Abuse, 36(5), 277-83. doi:10.3109/00952990.2010.491880

Wittek, C., Finserås, T., Pallesen, S., Mentzoni, R., Hanss, D., Griffiths, M., \& Molde, H. (2016). Prevalence and Predictors of Video Game Addiction: A Study Based on a National Representative Sample of Gamers. International journal of mental health and addiction, 14(5), 672-686. doi:10.1007/s11469-015-9592-8

World Health Organization. (2015, August 27-29). Public health implications of excessive use of the internet, computers, smartphones and similar electronic devices: meeting report, Main Meeting Hall, Foundation for Promotion of Cancer Research, National Cancer Research Centre, Tokyo, Japan. Retrieved December 2, 2020, from who.int: https://apps.who.int/iris/handle/10665/184264

World Health Organization. (2018, September 14). Addictive behaviours: Gaming disorder. Retrieved December 3, 2020, from https://www.who.int/news-room/q-a-detail/addictivebehaviours-gaming-disorder 